\title{
Le roi est mort, vive le roi?
}

\author{
Joachim Heinrich ${ }^{1,2}$
}

Affiliations: 'Ludwig Maximilians University Munich, University Hospital Munich, Institute and Outpatient Clinic for Occupational, Social and Environmental Medicine, Munich, Germany. ${ }^{2}$ Comprehensive Pneumology Center Munich (CPC-M), Member of the German Center for Lung Research, Munich, Germany.

Correspondence: Joachim Heinrich, Ludwig Maximilians University Munich, University Hospital Munich, Institute and Outpatient Clinic for Occupational, Social and Environmental Medicine, Ziemssenstrasse 1, 80336 München, Germany. E-mail: joachim.heinrichamed.uni-muenchen.de

@ERSpublications

Free sugar intake during pregnancy increases the risk of atopy in offspring, but replication is suggested http://ow.ly/TKIF30cy4Qo

Cite this article as: Heinrich J. Le roi est mort, vive le roi? Eur Respir J 2017; 50: 1701075 [https://doi.org/ 10.1183/13993003.01075-2017].

Barker's or Doerner's hypothesis on early programming factors during perinatal life leading to common chronic diseases $[1,2]$ is the scientific background for a massive amount of research on the potential impact of perinatal nutrition on the development of chronic diseases in later life. Pregnant women are extremely interested in what professionals state is the best nutritional care for a healthy baby. Journal editors and public media representatives are also very much engaged in the topic of perinatal nutrition, as it guarantees substantial attention from the scientific community and media customers. However, nutritional epidemiology has often been strongly attacked due to the observational nature of most of its studies and small randomised trials; but it has been particularly criticised for the limitations regarding dietary assessment, inconsistent findings and short-lived naive-driven recommendations [3]. Indeed, dietary recommendations for pregnant women have changed over the past decades, with the exception of folic acid and iodine intake, iron (in case of suboptimal iron stores) and gestational weight gain [4-8]. While the evidence of these recommendations in the prevention of neural tube defects or obesity-related disorders is sufficiently strong, there is only little evidence to support that specific dietary recommendations during pregnancy can prevent asthma and other allergic disorders. These recommendations include pre- or probiotics [9], as well as prenatal macronutrient and micronutrient intake. Historically, preventive recommendations focused on allergen avoidance in early life, but recent findings do not support this strategy or at least discuss the weaknesses of this approach [10], in particular for food allergies [11, 12]. Recent publications even reported that extended breastfeeding has no or only very little impact on the onset of asthma and other allergic diseases in childhood, although there are many other good reasons to recommend breastfeeding $[13,14]$. Thus, there is a strong need to understand the role of dietary intake during pregnancy with regard to developing allergies, specifically asthma, later in life. So far, observational studies in pregnant women explored the impact of allergenic foods, "healthy" food intake such as vegetables and fruit, the Mediterranean diet, and fatty acids, with mixed results. Dietary interventions in randomised control trials (RCTs) during pregnancy involving vitamin C supplementation, probiotics use, or avoidance of egg and cow's milk did not show any protective effects on allergies [15], whilst more recent RCTs showed borderline reduction of asthma for vitamin D supplementation during last trimester [16, 17], and a preventive potential of high fish oil supplementation during third trimester [18].

In this issue of the European Respiratory Journal, BÉDARD et al. [19] reported that a higher maternal intake of free sugar during pregnancy is associated with an increased risk of specific allergic sensitisation assessed by skin prick test and atopic asthma in children aged 7-9 years. This finding is novel and calls for further

Received: May 272017 | Accepted: June 022017

Conflict of interest: None declared.

Copyright @ERS 2017 
research, since it offers potential for new preventive measures. Data from 8956 subjects out of more than 14000 newborns recruited for the Avon Longitudinal Study of Parents and Children (ALSPAC; www. alspac.bris.ac.uk) were analysed. The dietary intake of mothers was assessed once at 32 weeks of gestation using a non-validated food frequency questionnaire (FFQ) "covering all the main foods consumed in Britain”, as the authors stated. Data on current weekly intakes of food groups and food items were collected and used to calculate total daily energy intake and nutrient intake. Free sugar intake was also estimated, not including lactose naturally present in milk and milk products, nor sugars contained within the cellular structure of fruits and vegetables. Thus, reported free sugar intake in the study includes sugars added to food or drinks by the manufacturer, cook or consumer and sugars naturally present in honey, syrups and unsweetened fruit juices. In addition, free sugar consumption of both the mother and father was assessed when the child reached an age of 4 years, and the free sugar consumption of the child was assessed at age 3 years. Data on respiratory health in terms of respiratory symptoms and doctor's diagnosis were collected, with strong time-point correlation. Further, a skin prick test against major common aeroallergens was performed at the age of 7 years. Asthma status was checked again at age 14 years in order to assess incident and remitting cases of asthmatics. Furthermore, spirometric lung function was tested and total IgE was measured in blood at age 7 years. Excellent statistical analyses and modelling were performed which made the most of the available data. The strongest association for free sugar intake during pregnancy with a clear exposure-response relationship was found for allergic sensitisation; and while the association with atopic asthma was also statistically significant, the linear increase with increasing free sugar intake in quintiles was not. Additional sensitivity analyses were thoughtfully performed, including adjustment for maternal weight characteristics to explore whether the reported association might be mediated by pre-pregnancy BMI, gestational age at delivery, birth weight, and child's BMI at the age of 7 years. None of these characteristics substantially changed the effect estimates. Therefore there is no indication that increased free sugar intake has an impact on allergic sensitisation via any altered weight characteristics of the mother or the child. In order to identify a "family effect", the paternal intake of free sugar was also analysed, but did not indicate any significant effect similar to maternal sugar intake. That result argue against unmeasured familial confounding. Finally, accounting for the child's sugar intake at age 3 years did not change the effect of maternal free sugar on allergic sensitisation. These sensitivity analyses were brilliantly performed and can defend against possible criticism of the study. A further major strength of this paper is the provided summary of potential underlying mechanisms for the reported association between free sugar intake and allergic sensitisation. The authors interpret the results to arise from high intakes of fructose among free sugars, which may cause the persistence of Th2 immune responses postnatally. This speculation seems reasonable, but needs to be explained by alternative and additional pathways. Although this study has several strengths there are also a number of limitations. There is a substantial proportion of loss to follow-up, but a differential misclassification and consequently bias of the results are not likely. In general, a FFQ is certainly not ideal to assess dietary intake on an individual basis, especially considering the low quality of the utilised FFQ with only very little validation activity. Free sugar intake in the case of the described study is more likely added sugar. Added sugar might be interpreted as a characteristic of dietary behaviour or "unhealthy" diet. Nevertheless, it is not clear whether this limitation in the assessment of free sugar intake would have produced an artificial association with allergic sensitisation. This study tested more than seven outcomes of interest, but showed strong and statistical significant associations only for allergic sensitisation, while the association with atopic asthma seems to be driven mainly by atopy. The authors did not adjust for multiple testing, but the exposure-response relationship for atopy was robust and would likely persist following correction for multiple testing.

It seems that more or less the same group of authors published four or five papers on potential associations between allergies and different maternal dietary components during pregnancy using mostly the same dietary dataset [20]. Unfortunately, the results are hard to compare due to methodological differences across these papers.

So far, more than 40 papers have been published on maternal diet and a broad spectrum of health outcomes using the data from ALSPAC. Thus, our current knowledge on health effects of maternal diet during pregnancy is largely influenced by this cohort. The impact of the dominance of just this one study is uncertain. On the other hand, one has to acknowledge the extremely high quality of the ALSPAC data and the availability of numerous unique variables.

The complexity of the diet makes it very difficult to suggest associations between its specific components and health outcomes of interest with certainty. Observational studies in nutrition regularly encounter problems, such as the limited validity of dietary assessment methods, as well as the correlation between different macro and micronutrients or residual confounding, which can never be entirely excluded. Observational studies claim to better cover real world scenarios of dietary intakes, but are often lacking 
specificity for certain dietary components. RCTs can better address the role of specific food items, but cannot completely solve the problems of uncertainty. Therefore, both observational studies and RCTs have their own specific advantages.

The authors of this study were wise in their interpretation of the findings, and hesitated to draw any conclusion in terms of dietary recommendation on free sugar intake during pregnancy to prevent allergic sensitisation. This would require an indication of causality in the reported association, which was not shown. Moreover, it is not entirely clear whether this stimulating association is more than just a chance finding. The authors have promised a replication of this study using the data from two other UK birth cohorts. Hence, until these findings are replicated, they can be viewed as nothing more than another episode in the chain of suggested maternal dietary factors impacting offspring allergies.

\section{References}

1 Barker DJ. Intrauterine programming of adult disease. Mol Med Today 1995; 1: 418-423.

2 Koletzko B. Developmental origins of adult disease: Barker's or Dorner's hypothesis? Am J Hum Biol 2005; 17: 381-382.

3 Satija A, Yu E, Willett WC, et al. Understanding nutritional epidemiology and its role in policy. Adv Nutr 2015; 6: 5-18.

4 Alavi N, Haley S, Chow K, et al. Comparison of national gestational weight gain guidelines and energy intake recommendations. Obes Rev 2013; 14: 68-85.

5 Chitayat D, Matsui D, Amitai Y, et al. Folic acid supplementation for pregnant women and those planning pregnancy: 2015 update. J Clin Pharmacol 2016; 56: 170-175.

6 Koletzko B, Bauer CP, Bung P, et al. German national consensus recommendations on nutrition and lifestyle in pregnancy by the 'Healthy Start - Young Family Network'. Ann Nutr Metab 2013; 63: 311-322.

7 Obeid R, Oexle K, Rissmann A, et al. Folate status and health: challenges and opportunities. J Perinat Med 2016; 44: $261-268$

8 World Health Organization. Guideline: intermittent iron and folic acid supplementation in non-anaemic pregnant women (in IRIS). Geneva, World Health Organization, 2011.

9 Cuello-Garcia CA, Brozek JL, Fiocchi A, et al. Probiotics for the prevention of allergy: A systematic review and meta-analysis of randomized controlled trials. J Allergy Clin Immunol 2015; 136: 952-961.

10 Heinrich J, Koletzko B, Koletzko S. Timing and diversity of complementary food introduction for prevention of allergic diseases. How early and how much? Expert Rev Clin Immunol 2014; 10: 701-704.

11 Du Toit G, Foong RM, Lack G. Prevention of food allergy - Early dietary interventions. Allergol Int 2016; 65: 370-377.

12 Togias A, Cooper SF, Acebal ML, et al. Addendum Guidelines for the Prevention of Peanut Allergy in the United States: Summary of the National Institute of Allergy and Infectious Diseases-Sponsored Expert Panel. J Acad Nutr Diet 2017; 117: 788-793.

13 Heinrich J. Modulation of allergy risk by breast feeding. Curr Opin Clin Nutr Metab Care 2017; 20: 217-221.

14 Victora CG, Bahl R, Barros AJ, et al. Breastfeeding in the 21st century: epidemiology, mechanisms, and lifelong effect. Lancet 2016; 387: 475-490.

15 Beasley R, Semprini A, Mitchell EA. Risk factors for asthma: is prevention possible? Lancet 2015; 386: 1075-1085.

16 Chawes BL, Bonnelykke K, Stokholm J, et al. Effect of vitamin D3 supplementation during pregnancy on risk of persistent wheeze in the offspring: a randomized clinical trial. JAMA 2016; 315: 353-361.

17 Litonjua AA, Carey VJ, Laranjo N, et al. Effect of prenatal supplementation with vitamin D on asthma or recurrent wheezing in offspring by age 3 years: The VDAART randomized clinical trial. JAMA 2016; 315: $362-370$.

18 Bisgaard H, Stokholm J, Chawes BL, et al. Fish oil-derived fatty acids in pregnancy and wheeze and asthma in offspring. N Engl J Med 2016; 375: 2530-2539.

19 Bédard A, Northstone K, Henderson AJ, et al. Maternal sugar intake during pregnancy and childhood respiratory and atopic outcomes. Eur Respir J 2016; 50: 1700073.

20 Emmett PM, Jones LR, Golding J. Pregnancy diet and associated outcomes in the Avon Longitudinal Study of Parents and Children. Nutr Rev 2015; 73: Suppl. 3, 154-174. 